\title{
Elastography. higher quality and confidence for better characterization of musculoskeletal injuries
}

\begin{abstract}
The musculoskeletal injuries are common in units of pain and often have difficulties in their final resolution. The appearance of elastography represents a quantum leap of extraordinary value, ensures a better assessment of the injury, which may have predicted areas injury in the immediate future, the evolution of our valued patients and more reliable manner is a great support to those who we are dedicated to interventionism. We present the case of a 36 year old male, (professional golfer) diagnosed with Elbow Golfer. The use of ultrasound with elastography allowed us to improve access to the injured area, monitor your progress, and to determine the optimal time to define the total resolution of the damage.
\end{abstract}

Keywords: Elastography, Elasticity imaging, Chronic pain, Musculoskeletal injuries, Musculoskeletal alterations, Sonoelastography, Ultrasound
Volume I Issue I - 2014

\section{Ernesto delgado cidranes}

Department of Anesthesiology and Pain Medicine, Ruber Clinic, Spain

Correspondence: Ernesto Delgado Cidranes, Departament of Anesthesiology and Pain Medicine, Ruber Clinic of Madrid, Juan Bravo 49, CP 28006, Spain, Tel 0034-9I-I II-930I,

Email edelgadocidranes@gmaill.com

Received: May 04, 2014 | Published: May II, 2014

\section{Introduction}

The appearance of elastography represents a quantum leap of extraordinary value. ${ }^{1}$ Spin and interventionism "blind" is somewhat archaic, obsolete in a technological world where ultrasound and occupies an important place. The good practice passes properly assess an injury to the patient and provide the best solution. In recent years elastography has provided a better characterization of musculoskeletal injuries. The patient is a participant in the proceeding, look at where the problem is, the treatment is observed and evaluated by the professional evolution. Ultrasound elastography using quantitative strain ratio measurements could be a promising supplementary method to evaluate tendon abnormalities. ${ }^{2}$

\section{Case report}

In our pain unit of the Ruber Clinic in Madrid received a 36 year old male professional golfer who suffered from about two years ago a history of pain in the inside of his right elbow, with progressive worsening in the last two months we causing severe pain and loss of function of the affected limb. He had been diagnosed with Tennis Elbow and received multiple treatments but each time restarting play again and fall injury and injury chronified. Before starting new cycle of intervention is decided to perform assessment in consultation with elastography. B-mode images were obtained, with and without Doppler and subsequently for elastography assessment was performed. Clinical and ultrasonographic data obtained with different methods and strategic behavior discussed to follow based on information provided by the elastic elastography were compared. We note that the thickening of the tendon and hypoechoic structure was present in B-mode but applying elastography find all the very tender area, predominantly red showing loss of normal tendon stiffness (Figure 1). Treatment and follow-up to final evaluation was formalized. A total of four sessions of ultrasound-guided intervention and assessment were planned under elastográfica. The sonographic improvement was evident from the ssecond session. We apply Ozone treatment and platelet growth factors.

\section{Discussion}

Basically it is to know how a human tissue is elastic, as if the browser could touch it with their hands. Depending on the elasticity obtain important information about the nature of the court explored. When a patient presents an accessible manual palpation tumor and the browser sees with his hands how the fabric is to be tough, inelastic and adhered to deep planes, this immediately raises the possibility that what feels to be malignant. The idea elastography is to "feel" an inaccessible tissue, and thus be able to study the nature of a lump or mass suspicious for malignancy in its early stages and thus guide the diagnosis avoiding more expensive tests or speeding timeouts.

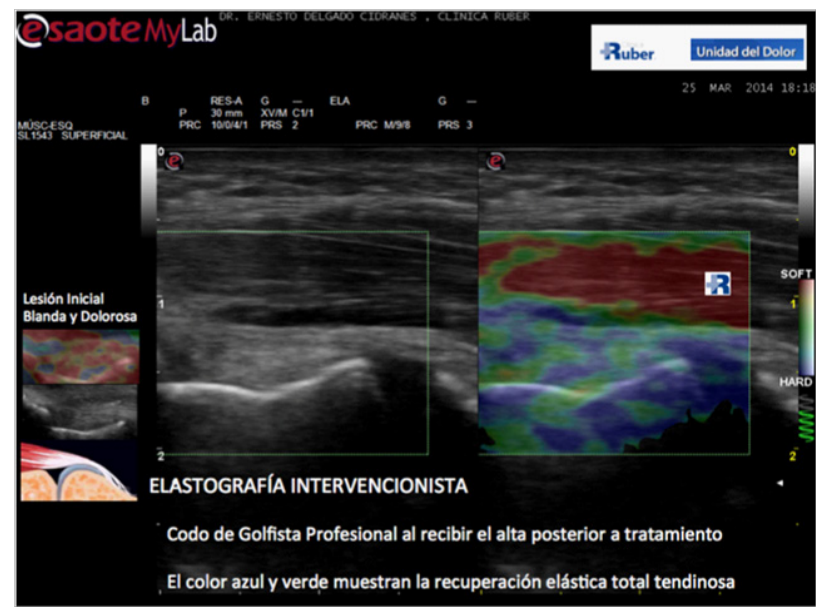

Figure I Elastography Interventional. Professional Golfer Elbow at discharge after treatment. Blue and green show the tendon elastic recovery. The soft and painful initial lesion is shown in red on the small image to the left.

The technique is applicable to all tissues, not just as a tool to look for cancer, but elastography primarily intended to be a useful resource in the diagnosis of cancer. It has been seen that the combined elastography with ultrasound and mammography increases diagnostic performance in breast cancer. In thyroid, pancreas, prostate and lymph nodes are studying their diagnostic possibilities. Liver Elastography was the first to be carried out and proved to be where the first positive results were seen as a technique for assessing diffuse liver disease and cirrhosis. It is showing good results in the study of the blood vessel wall, thrombotic disease, heart tissue and musculesquelétic system. Muscle architecture can be measured concurrently with muscle hardness using ultrasound strain elastography. ${ }^{3}$ 
The image that the sonographer obtained on the monitor in color, can discern more elastic tissues less elastic with a glance. The possibilities are not negligible because by elastography may be possible to orient the focus punctures and biopsies of benign or malignant lesion, can detail the extent of the lesion by U.S. and might even consider monitoring the effectiveness of treatment, among other things. Elastography provide a biomechanical explanation for the observed during rehabilitation in clinical practice effect. ${ }^{4}$ It is very useful to suggest the consistency of a scar after a break of fibers in a muscle, tendon or ligament and to advise whether or not you can return to sports, work, etc.

\section{Conclusion}

We present a great option to improve the assessment, treatment and monitoring of injury musculoesquleticas until full resolution. The B (in black and white Ultrasound) mode provides an acceptable, feasible diagnostic information but when combined with elastography can better quantify and qualify the severity of the injury and its progress in just as treatment is received. ${ }^{5}$

\section{Acknowledgments}

None.

\section{Conflicts of interset}

None.

\section{References}

1. Ophir J, Cespedes I, Ponnekanti H, et al. Elastography: a quantitative method for imaging the elasticity of biological tissues. Ultrason Imaging. 1991;13(2):111-134.

2. Ahn KS, Kang $\mathrm{CH}$, Hong SJ, et al. Ultrasound elastography of lateral epicondylosis: clinical feasibility of quantitative elastographic measurements. AJR Am J Roentgenol. 2014;202(5):1094-1099.

3. Chino K, Akagi R, Dohi M, et al. Measurement of muscle architecture concurrently with muscle hardness using ultrasound strain elastography. Acta Radiol. 2013;[Epub ahead of print]

4. Hug F, Ouellette A, Vicenzino B, et al. Deloading tape reduces muscle stress at rest and during contraction. Med Sci Sports Exerc. 2014;[Epub ahead of print].

5. Porta F, Damjanov N, Galluccio F, et al. Ultrasound elastography is a reproducible and feasible tool for the evaluation of the patellar tendon in healthy subjects. Int J Rheum Dis. 2013;doi: 10.1111/1756-185X.12241. 